\title{
Identification of an Embryonic Cell-Specific Region within the Pineapple SERK1 Promoter
}

\author{
Aiping Luan ${ }^{1,2}$, Yehua He ${ }^{2, *}$, Tao Xie ${ }^{2}$, Chengjie Chen ${ }^{2}$, Qi Mao ${ }^{2,3}$, Xiaoshuang Wang ${ }^{2}$, \\ Chuhao Li ${ }^{2}$, Yaqi Ding ${ }^{2}$, Wenqiu Lin ${ }^{2}$, Chaoyang Liu ${ }^{2}$, Jingxian Xia ${ }^{2}$ and Junhu He ${ }^{1}$ \\ 1 Tropical Crops Genetic Resources Institute of Chinese Academy of Tropical Agricultural Science, \\ Haikou 571101, China; aipingluan@hotmail.com (A.L.); hejunhu123@163.com (J.H.) \\ 2 College of Horticulture, South China Agricultural University, Guangzhou 510642, China; \\ xietao@fosu.edu.cn (T.X.); ccj0410@gmail.com (C.C.); zjmaoqi@163.com (Q.M.); \\ Luckygirlkeepsmile@163.com (X.W.); lichuhao@scau.edu.cn (C.L.); dyqkxy2019@126.com (Y.D.); \\ linwenqiu1989@163.com (W.L.); liuchaoyang@scau.edu.cn (C.L.); xjx0036@scau.edu.cn (J.X.) \\ 3 College of Agricultural, Guangdong Ocean University, Zhanjiang 524088, China \\ * Correspondence: heyehua@hotmail.com; Tel.: +86-020-8528-8262
}

Received: 14 October 2019; Accepted: 29 October 2019; Published: 1 November 2019

\begin{abstract}
Plant tissue culture methods, such as somatic embryogenesis, are attractive alternatives to traditional breeding methods for plant propagation. However, they often suffer from limited efficiency. Somatic embryogenesis receptor kinase (SERK)1 is a marker gene of early somatic embryogenesis in several plants, including pineapple. It can be selectively induced and promotes a key step in somatic embryogenesis. We investigated the embryonic cell-specific transcriptional regulation of AcSERK1 by constructing a series of vectors carrying the GUS (Beta-glucuronidase) reporter gene under the control of different candidate cis-regulatory sequences. These vectors were transfected into both embryonic and non-embryonic callus, and three immature embryo stages and the embryonic-specific activity of the promoter fragments was analyzed. We found that the activity of the regulatory sequence of AcSERK1 lacking $-983 \mathrm{nt}-880 \mathrm{nt}$, which included the transcription initiation site, was significantly reduced in the embryonic callus of pineapple, accompanied by the loss of embryonic cell-specific promoter activity. Thus, this fragment is an essential functional segment with highly specific promoter activity for embryonic cells, and it is active only from the early stages of somatic embryo development to the globular embryo stage. This study lays the foundation for identifying mechanisms that enhance the efficiency of somatic embryogenesis in pineapple and other plants.
\end{abstract}

Keywords: pineapple; AcSERK1; embryonic cell-specific promoter; regulatory sequences; somatic embryogenesis

\section{Introduction}

The pineapple (Ananas comosus L.) is a perennial herb from the Bromeliaceae family and one of the world's most important tropical fruit species. Pineapple is largely vegetatively propagated because the species is self-sterile. This has traditionally involved sucker propagation, which is a simple and low-cost process. However, it suffers from several significant limitations, including a low reproductive coefficient, prolonged production periods, and non-uniform growth and development [1]. To avoid these problems, pineapple breeding by tissue culture has been developed as an attractive alternative to traditional breeding methods. This approach has several key advantages, including rapid and uniform growth and development of plants, a two-month reduction in production cycle, and viral disease reduction.

Organogenesis and somatic embryogenesis are the two in vitro tissue culture techniques used for plant regeneration, and both have been applied to the pineapple [1-4]. However, unlike organogenesis, 
somatic embryogenesis (SE) is an ideal rapid propagation method for many different types of in vitro plants and trees and fits the requirements of industrialization [5]. The transition from somatic cell to embryonic cells is a key stage in SE, and appears to be a critical step limiting efficiency for plant regeneration [6-8]. Thus, understanding the mechanisms involved in the somatic to embryonic cell transition in order to improve SE efficiency has become a major focus in the field [9].

The SE pathway in animals and plants is regulated by a variety of factors [10]. Somatic embryogenesis receptor kinases (SERKS) are a small gene family of receptor-like kinases that play diverse roles in plants, including in SE, pluripotency, reproductive development, the immune response, and stomatal patterning [11,12]. According to the NCBI database, SERK genes have been found, thus far, in 49 species. Most of the SERK genes from different species share a similar gene structure [13]. Among the SERK family, SERK1 is regarded as an SE marker gene of pineapple and other plants [14,15]. SERK1 expression is associated with induction of SE and also promotes the transformation of somatic cells to embryonic cells. For instance, expression of SERK1 in Arabidopsis thaliana was shown to increase its SE capacity by three to four times [16]. SERK1 expression can be induced by plant hormones, such as 6-BA, NAA [17], and 2,4-D [18], and by disease defense signaling molecules, such as SA, $\mathrm{BTH}, \mathrm{JA}$, and ABA [14,19]. This would suggest that its regulatory sequences/promoter contains a corresponding cis-acting element that responds to the induction factors [20-25]. While the SERK1 gene shows enhanced expression in embryogenic cells in many other plant species upon treatment with plant growth regulators, it is normally also expressed in a subset of somatic cells. In Arabidopsis thaliana, AtSERK1 is first expressed in the developing ovule [16]. In maize, ZmSERK1 is preferentially expressed in male and female reproductive tissues and is most strongly expressed in microspores [26]. In rice, OsSERK1 promote the differentiation of rice callus into adventitious buds [19] and is likely to play a more prominent role in non-embryonic tissues [27]. The expression of SERK during somatic embryogenesis of potato revealed that it increased during induction. There was no change in StSERK gene expression during subsequent embryonic development and embryo maturation, and StSERK had different levels of expression in other plant organs (leaves, seeds, tubers, and flower buds) [28]. These findings indicate that SERK1 predicts embryogenic potential but is not exclusively associated with early embryogenesis.

In our previous study, we cloned three SERK genes from pineapple [14,29]. Subsequent bioinformatics analysis indicated that they belonged to the leucine-rich repeat receptor-like kinase (LRR-RLK) gene family and possessed the characteristic conserved domain and the conserved exon / intron structure shared by the SERK gene family. Only very low expression levels of the three AcSERKS could be detected in non-embryonic callus, roots, stems, leaves, calyx, bracts, petals, anthers, ovary and ovules, parenchyma cells in young stems, parenchyma cells in the stem cortex, meristematic cells in roots, and unexpanded young leaves. Expression of all three AcSERKs could be induced by mechanical injury, salicylic acid, methyl jasmonate, and high salt treatment. In addition, unlike AcSERK3, expression of AcSERK1 and AcSERK2 could also be induced by low temperature. The hybrid signal of AcSERK1 appeared strongly in single embryonic cells, then gradually weakened, remaining detectable till the early globular stage of somatic embryos, and then further decreased in the late globular stage [14,29]. According to AcSERK1 expression characteristics, an embryonic cell-specific region was predicted to be present in its regulatory sequence.

In order to study the transcriptional regulation mechanism of AcSERK1, we previously cloned the promoter of AcSERK1. We found that the TSS (Transcription Start Sites) (+1) was the 258th nucleotide (G) upstream from the ATG (initiation codon), the length of the general promoter region was $2090 \mathrm{bp}$, and the length of the $5^{\prime}$-UTR was $258 \mathrm{bp}(+1 \sim+258)$. A recombinant vector consisting of the AcSERK1 $5^{\prime}$-upstream region $(2090 \sim+258)$ and the reporter gene GUS was constructed to enable transient transformation into different organs, and the embryonic and non-embryonic callus of pineapple, to analyze its expression patterns. Subsequent histochemical staining showed no obvious staining in the leaves, stems, roots, anthers, petals, ovary, and non-embryonic callus. Notably, GUS staining was observed only in 2,4-D-induced embryonic callus. This indicates that the complete regulatory sequence 
in the $5^{\prime}$-upstream region of AcSERK1 had no activity in non-embryonic cells and, therefore, that its promoter activity was specific to embryonic cells [30]. In this study, we constructed a series of deletion vectors with GUS as a reporter gene, which were constructed to further isolate and characterize the embryonic cell-specific region.

\section{Materials and Methods}

\subsection{Vector Construction}

Using GUS as a reporter gene, a series of recombinant expression vectors containing the AcSERK1 upstream regulatory sequences with different deletions (Figure S1) were constructed, with reference to the pAS2 $(-2090 \sim+258)$ :: GUS recombinant vector construction procedure [30]. Briefly, this procedure involved replacing the CaMV 35S promoter in the binary expression vector pBI121 using the XbaI and HindIII sites with the complete $5^{\prime}$ upstream regulatory sequence of AcSERK1 by homologous recombination with the primers AS1-F (TGATTACGCCAAGCTTATAAATAATTAGACACTTCACGCAAC), and AS1-R (CCGGGGATCCTCTAGATGCCGCCGCCGCGAGCT). The position of cis elements in the AcSERK1 upstream regulatory sequences was predicted using the signal scanning function of the PlantCARE database [31]. Subsequently, specific primers were designed to avoid destroying the predicted cis-acting elements and various deletion regions were amplified by PCR (primers shown in Figure S1). Primers were designed to introduce $15 \mathrm{bp}$ homologous sequences on both sides of the XbaI and HindIII cleavage sites at the $5^{\prime}$ and $3^{\prime}$ ends of the promoter deletion region. The vector was linearized with the restriction enzymes XbaI and HindIII, and construction of the recombinant plasmids was completed by homologous recombination of the linearized vector and the amplified promoter deletion regions using In-Fusion enzyme (TaKaRa, Dalian China). Next, the plasmid was transformed into Escherichia coli $\mathrm{DH} 5 \alpha$ strain by heat shock, and positive clones were identified by sequencing. The plasmids extracted from the positive clones were transfected into Agrobacterium tumefaciens GV3101 by heat shock. All PCR amplified DNA fragments were verified by sequencing.

\subsection{Plant Materials}

The pineapple species, 'Shenwan' (Ananas comosus L.), used was collected from the garden of South China Agricultural University in Guangzhou, China, in 2013. Suckers were obtained from the pineapple and cultured in embryonic induction medium (Murashige and Skoog [MS] + $5 \mathrm{mg} / \mathrm{L} \mathrm{2,4-D} \mathrm{+}$ $0.5 \mathrm{mg} / \mathrm{L} \mathrm{BA}$ ) to induce embryonic callus, or in medium without 2,4-D (MS + $2 \mathrm{mg} / \mathrm{L} \mathrm{NAA}+3 \mathrm{mg} / \mathrm{L}$ BA) for non-embryonic callus [32]. The culture obtained by induction of somatic embryos for 40 days (embryonic callus mainly containing globular embryo) was transferred to somatic embryo development medium (MS + $1 \mathrm{mg} / \mathrm{L} \mathrm{NAA}+0.5 \mathrm{mg} / \mathrm{L} \mathrm{BA}$ ) for 10 days (mainly developing into pyriform embryo) and 20 days (mainly developing into bamboo shoot embryo). The embryonic and non-embryonic callus and immature embryos (globular embryo, pyriform embryo, and bamboo shoot embryo) were then used for transient transfection experiments (Section 2.3).

\subsection{Transient Transfection}

Callus was collected after 5, 10, 15, 20, 25, 30, 35, 40, 45, and 50 days of culture in embryonic induction medium, or after culture in non-embryonic induction medium (embryonic callus and non-embryonic callus, respectively) for transient transformation. Callus for observation of promoter activity in immature embryos was collected after being cultured for 10 days and 20 days in somatic embryo development medium (see above). The collected callus were cut into 3 $33 \times 3 \mathrm{~mm}$ cubes for immediate Agrobacterium-mediated transient transformation. Briefly, the transfected Agrobacterium tumefaciens GV3101 was cultured by streaking, and single colonies were picked and inoculated into a YEB (Yeast Mannitol Medium) liquid medium and cultured overnight at $28^{\circ} \mathrm{C}$ with shaking. Once the concentration reached an absorbance value of $\sim 0.5$ at $600 \mathrm{~nm}\left(\mathrm{OD}_{600}=0.5\right)$, 
the Agrobacterium solution was used for the transient transformation experiments. In order to eliminate the error caused by different conversion efficiencies and protein extraction efficiencies between different batches, an internal standard vector (CaMV 35S :: Fluc) was introduced with the test vector [33]. Note that the test vectors and the internal standard vectors were prepared separately. The final mixture was prepared with a ratio of test carrier: internal standard carrier $=2: 1(v: v)$. Next, the pineapple callus samples were infected with this mixture using vacuum infiltration [34]. After infection, the callus samples were cultured in the dark on medium containing $\mathrm{MS}+2 \mathrm{mg} / \mathrm{L} \mathrm{NAA}+3 \mathrm{mg} / \mathrm{L} \mathrm{BA}$ for two days. Subsequently, 10 pieces of callus from each material transformed with a different deletion expression vector were collected for GUS histochemical staining. In addition, $\sim 1 \mathrm{~g}$ of each material was immediately fixed with liquid nitrogen and stored at $-80{ }^{\circ} \mathrm{C}$ for quantitative assays (Section 2.4).

\subsection{GUS Histochemical Staining}

Ten pieces of callus for GUS histochemical staining were immersed in X-Gluc staining solution (1 mM X-Gluc, $0.1 \mathrm{M}$ sodium phosphate buffer $\mathrm{pH}$ 7.0, 0.1\% Triton X-100, $8 \mathrm{mM}$-mercaptoethanol) and incubated at $37^{\circ} \mathrm{C}$ in the dark for $16 \mathrm{~h}$. After triplicate decolorization by $75 \%$ ethanol at $65{ }^{\circ} \mathrm{C}$ for $30 \mathrm{~min}$, samples were analyzed using an Olympus stereo microscope.

\subsection{Promoter Activity Assay}

The quantitative promoter activity assays using GUS fluorescence in embryonic versus non-embryonic callus were performed using methods described previously $[33,35]$. Briefly, the callus stored at $-80^{\circ} \mathrm{C}$ was ground with liquid nitrogen, and $100 \mathrm{mg}$ of the sample powder was added to $400 \mu \mathrm{L}$ of cell culture lysis reagent (CCLR, Promega, Madison, WI, USA). After mixing, the mixture was put in an ice bath for $10 \mathrm{~min}$, and then centrifuged at $13,000 \times g$ for $5 \mathrm{~min}$. The supernatant was dispensed into a new centrifuge tube for GUS and Fluc (Firefly Luciferase gene, internal control) assays. For the GUS assay, $20 \mu \mathrm{L}$ supernatant was taken and immediately mixed with $480 \mu \mathrm{L}$ reaction solution (0.1M sodium phosphate buffer $\mathrm{pH} 7.0,0.5 \mathrm{M}$ EDTA pH 8.0, 0.1\% Triton X-100, 10mM-mercaptoethanol, $0.1 \%$ sodium N-laurosylsarcosine, $1 \mathrm{mM}$ 4-Methylumbelliferyl-b-d-glucuronide), and incubated in a $37^{\circ} \mathrm{C}$ water bath. One hundred microliters of the mixture was taken at $0 \mathrm{~min}$ and $30 \mathrm{~min}$, and $900 \mu \mathrm{L}$ of $200 \mathrm{mM}$ sodium carbonate buffer was added to each sample to terminate the reaction. After mixing, $200 \mu \mathrm{L}$ of sample was taken and measured using the Hitachi F-4600 Fluorescence Spectrophotometer (Tokyo, Japan) with the emission light set to $465 \mathrm{~nm}$ and the excitation light set to $340 \mathrm{~nm}$. A GUS activity unit is nmol 4-methyl umbelliferone (4-MU) minute ${ }^{-1}$. The detection of the luminous value of Fluc was carried out following the instructions of the Promifer Luciferase Assay System (E1500). Thermo Scientific Luminoskan Ascent (Waltham, MA, USA) was used, with $10 \mathrm{~s}$ as the reading time.

The fluorescence values of GUS and the luminescence value of Fluc were detected separately, and the ratio of the two tests was set as the initial value of the promoter activity. Note that the initial value unit was nmol 4-MU min $^{-1}$ per light units/10 s. To determine embryonic cell-specific promoter activity, we used the ratio of the initial value of the promoter activity in the embryonic tissue to that in the non-embryonic tissue. A relative promoter activity of 1 refers to roughly equal promoter activity in both embryonic and non-embryonic callus.

\subsection{Gene Expression Analysis}

Total RNA from each callus sample was extracted using TRIzol reagent (Invitrogen, Shanghai, China) and cDNAs were synthesized using PrimeScript ${ }^{\mathrm{TM}}$ RT reagent Kit with gDNA Eraser (TaKaRa, Dalian, China). Quantitative real-time PCR (qPCR) was performed by Thunderbird SYBR qPCR Mix(Toyobo, Shanghai, China)in the iQ5 Real-time PCR system (BioRad, Hercules, CA, USA). The primers used are listed in Table 1 . The specificity of primers was confirmed by melting curve analysis. Each reaction was performed in biological triplicates, and the relative gene expression values were calculated using the $2^{-\triangle \Delta C T}$ method. The expression levels were normalized against the pineapple $\beta$-actin gene [14]. 
Table 1. Primers used for qPCR.

\begin{tabular}{ccc}
\hline Primer Name & Primer Sequence & References \\
\hline GUS $_{\mathrm{F}}$ & $5^{\prime}$-AACCGTTCTACTTTACTGGCTTTGG-3' & Wang et al., 2013 [36] \\
GUS $_{\mathrm{R}}$ & $5^{\prime}$-GCATCTCTTCAGCGTAAGGGTAAT-3' & \\
Fluc-F $_{\text {Fluc- }_{\mathrm{R}}}$ & $5^{\prime}$-TGCACATATCGAGGTGGACATC-3' & Murray et al., 2017 [37] \\
5-Actin $_{\mathrm{F}}$ & $5^{\prime}$-CTGACGCCAGAAGAATTTGCAGCAT-3' & \\
$\beta$-actin & 5'CACTTCTGGGCAGCGCACTTGACTT-3' $_{\mathrm{R}}^{\prime}$ & Ma et al., 2012 [14] \\
\hline
\end{tabular}

\section{Results}

\subsection{Analysis of the Promoter Activity of the Complete 5' Upstream Regulatory Sequence of AcSERK1 during} the Induction of Pineapple SE

The transition from somatic cell to embryonic cell is one of the key stages limiting SE efficiency for plant regeneration [6-8]. SERK1 is known to promote SE, and we previously identified promoter activity in the complete $5^{\prime}$ upstream regulatory sequence $(-2090 \sim+258)$ of AcSERK1 that was specific to embryonic cells [30]. We first sought to further dissect this regulatory sequence and identify the region required for its activity during SE. We generated embryonic callus by culturing suckers in embryonic induction medium (containing $\mathrm{MS}+5 \mathrm{mg} / \mathrm{L} \mathrm{2,4-D}+0.5 \mathrm{mg} / \mathrm{L} \mathrm{BA}$, see methods) for 0 (i.e., non-embryonic callus), 10, 20, 30, 40, and 50 days. The callus was then collected at each timepoint and used for Agrobacterium-mediated transient transformation with a recombinant expression vector containing the previously isolated AcSERK1 regulatory sequence upstream of the GUS reporter gene (pAS2 [-2090 +258] :: GUS reporter), and incubated for two days. In callus cultured for only 10 days in embryonic induction medium, weak GUS histochemical staining was detectable over a very small surface area of the callus (Figure 1A). In contrast, callus collected after 20 days of induction displayed more extensive GUS staining, which increased in area and intensity in callus collected after 30 days of induction. The deepest staining and largest stained area was observed in callus after 40 days of induction (Figure 1A, indicated by arrows), which dramatically decreased after 50 days. As a negative control, we transfected the pAS2 [-2090 +258] :: GUS reporter into plants grown from somatic embryos and observed no staining. Thus, SERK1 promoter activity increases over the course of SE, before subsequently decreasing.

We next quantified the activity of AcSERK1 during SE induction by quantifying the expression levels of the GUS gene using qPCR analysis. qPCR of the co-transformed CaMV $35 \mathrm{~S}$ promoter upstream of the Fluc gene was used as a control for transformation efficiency (see methods). Consistent with the data above, the expression of the pAS2 (-2090 +258) :: GUS reporter first increased up to 40 days induction, and then decreased, while Fluc expression remained relatively constant (Figure 1B). To measure the embryonic-specific promoter activity of pAS2 $(-2090 \sim+258)$ during SE induction at the protein level, we quantified GUS fluorescence levels in the embryonic vs non-embryonic tissues using spectrophotometry (see methods). A relative promoter activity value of 1 indicates equal promoter activity in both embryonic and non-embryonic callus. Under the same transformation conditions, we found that the relative promoter activity of pAS2 remained fairly constant between 0 and 10 days of induction (Figure 1C), then gradually increased reaching a peak on day 40 at 26 times the levels in non-embryonic tissues. Finally, there was a significant decrease to three times non-embryonic levels. Thus, promoter activity monitored at the expression level followed a similar trend to the GUS qPCR assay result. Together, these results further support a role for AcSERK1 specifically in SE and reveal the dynamics of promoter activity over the course of SE. 

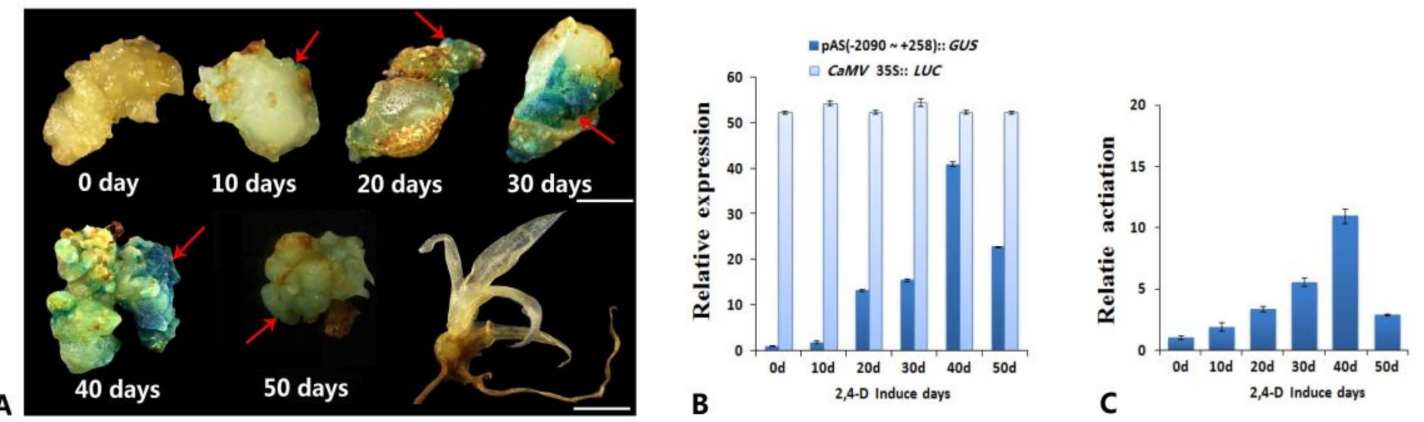

Figure 1. Transient expression analysis of promoter activity of the $5^{\prime}$ upstream regulatory sequence of AcSERK1. Callus was isolated after different lengths of time cultured in embryonic induction medium and analyzed by GUS histochemical staining. Red arrows indicate areas of GUS expression: (A) 0 days (d, i.e., non-embryonic callus), 10 days, 20 days, 30 days, 40 days, 50 days, and plant regenerated from somatic embryo (negative control). Scale bar of callus indicates $2 \mathrm{~mm}$, Scale bar of plant regenerated from somatic embryo indicates $5 \mathrm{~mm}$. (B) Relative expression levels of the GUS gene measured by qPCR during the induction of pineapple SE(somatic embryogenesis). Pineapple $\beta$-actin gene served as the internal control. Data represent three biological replicates and error bars denote standard error of the mean. (C) Quantitative GUS assay to determine embryonic specific promoter activity. Data plotted as relative promoter activity: a value of 1 indicates equal promoter activity in both embryonic and non-embryonic callus. Data represent three biological replicates, and the error bars denote standard error of the mean.

\subsection{Identification of the Embryonic Cell-Specific Region in the 5' Upstream Regulatory Region of AcSERK1}

Given the strongest activity of the $5^{\prime}$ upstream regulatory sequence of AcSERK1 was observed in callus after 40 days of SE induction by 2,4-D, we used this material for our deletion analysis. We generated a series of 12 deletion mutants of the promoter, working around predicted cis regulatory sequences (see Methods and Figure S1). Data from only a selection of this series needed to pinpoint the active promoter region are shown. In embryonic callus transformed with the deletion mutant pAS4 $(-1138 \sim+258)::$ GUS, and the positive control, CaMV 35S :: GUS, we observed strong GUS histochemical staining (Figure 2A). However, embryonic callus transformed with pAS5 $(-772 \sim+258)::$ GUS in which the deletion further expanded to $772 \mathrm{nt}$ upstream relative to the TSS was stained only faintly. Thus, the promoter activity is located between -1138 and -772 . Further expression vectors were designed to span this region. Almost no staining was observed in embryonic callus after transient transformation with the recombinant expression vector pAS15 carrying a deletion of $(-983 /-880)$, whereas intensive staining was observed with pAS16: the $(-880 /-772)$ deletion (Figure 2A). Only very faint staining was observed in embryonic callus transformed with the negative control pCK (GUS-nos) (Figure 2A).

We next quantified the relative promoter activities as above of the different mutants, which are shown in Figure 2B. When deletion in the regulatory sequence expanded to $772 \mathrm{nt}$ upstream relative to TSS (pAS5), its relative promoter activity was 1 (i.e., equal in both embryonic and non-embryonic callus). In contrast, when the deletion of the regulatory sequence was set only to $-1138 \mathrm{nt}$, the relative activity of pAS4 $(-1138 \sim+258)$ significantly increased, reaching 37.1 . The relative promoter activity of the regulatory sequence with deletion of $(-880 /-772)$ was 37.0 (pAS16), whereas the middle deletion of $(-983 /-880)$ was 1.1 (pAS15). Thus, consistent with the GUS staining results, these data indicate that deletion of (-983/-880) directly affects the embryonic cell-specific promoter activity of the AcSERK1 regulatory sequence. 

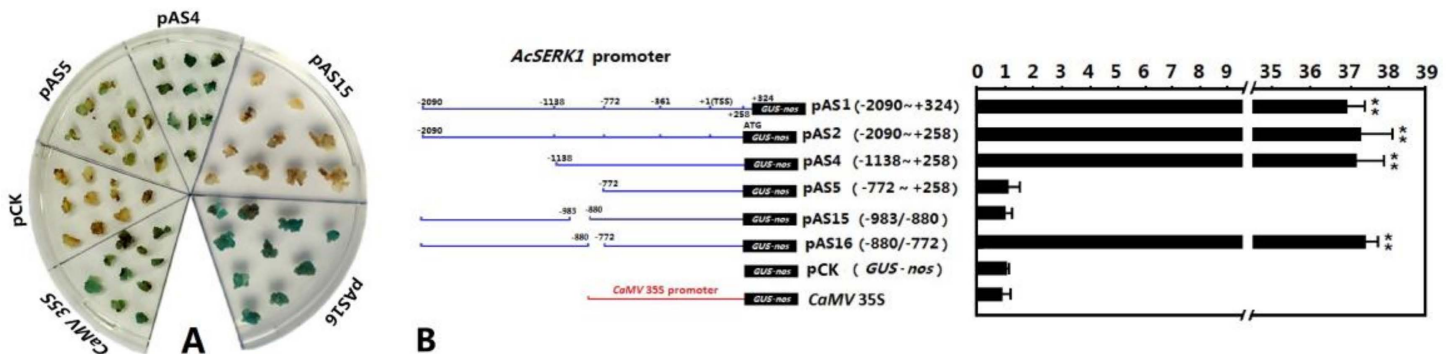

Figure 2. Deletion analysis of the embryonic cell-specific region in the $5^{\prime}$ upstream regulatory region of AcSERK1. (A) The results of GUS staining in the embryonic callus, (B) The quantitative measurements of promoter activity. The numbers in the vector map (left) indicate the deletion position. The vectors were co-infected with the internal standard vector CaMV 35S :: Fluc (pBI121-Fluc) into pineapple callus (embryonic and non-embryonic). The bars represent relative promoter activities (the ratio of GUS activity to Fluc activity) in the embryonic callus to those of the non-embryonic callus (i.e., embryo-specific promoter activity). The data represent the average of three biological replicates, and the error bars denote Standard Deviation (SD). ${ }^{* *}$ highly significant $(p<0.01)$, one-tailed $t$-test.

We further analyzed three immature embryo stages by GUS staining. The stages of the immature embryo in pineapple include the globular embryo, pyriform embryo, and bamboo shoot embryo stages, similar to the development of the zygotic embryo. The globular embryo was transformed with the pAS2 (-2090 +258) :: GUS reporter, and we observed strong GUS histochemical staining (Figure 3A). However, almost no staining was observed in the pyriform embryo or the bamboo shoot embryo after transient transformation with pAS2 (Figure 3B,C). In the three immature embryo stages respectively transformed with the pAS15 vector carrying a deletion of (-983/-880), we also observed almost no GUS histochemical staining (Figure 3D,E,F). These GUS staining results of the globular embryo stage again confirmed that deletion of $(-983 /-880)$ directly affects the embryonic cell-specific promoter activity of the AcSERK1 regulatory sequence.

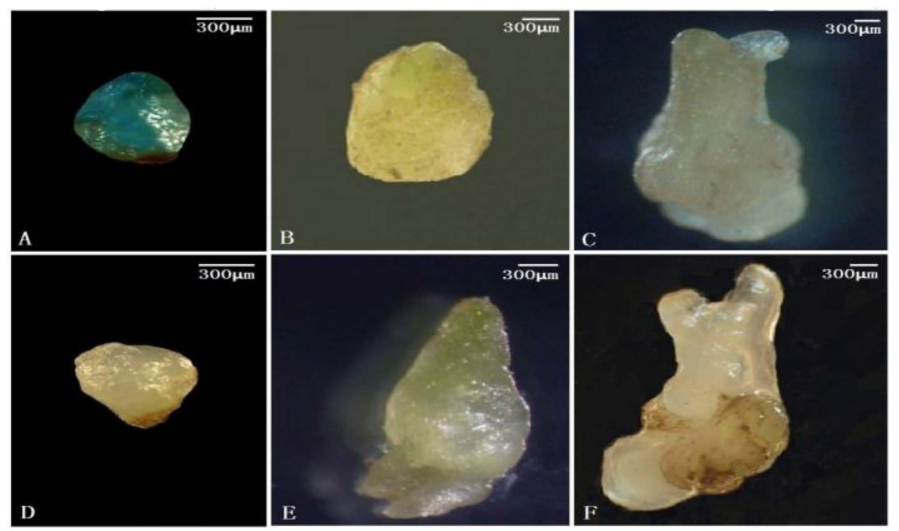

Figure 3. Transient expression analysis of promoter activity with pAS2 $(-2090 \sim+258)::$ GUS and pAS5 $(-772 \sim+258)::$ GUS in three stages of immature embryos.

\subsection{Bioinformatics Analysis}

The 103 bp region between $-983 \mathrm{nt}$ and $-880 \mathrm{nt}$ was predicted by bioinformatics analysis (see methods) to contain only three CAAT-box elements (-CAAAT, +CAAT, and -CAAT, where "+" "-" indicate coding or noncoding chains) (Figure 4). In addition, NCBI blast of the 103 bp region in the AcSERK1 promoter with the $5^{\prime}$ upstream regulatory sequence of AcSERK2 and AcSERK3, revealed only 11 bp of homologous sequence (sequence information is indicated by arrows in Figure 4) with AcSERK3, and no homologous sequence (length $>4 \mathrm{bp}$ ) with $A c S E R K 2$. This provides further support of an embryonic-specific function for AcSERK1. In green plants, 40 coding genes (Figure S2) associated with 
somatic embryogenesis from the SERK family can be retrieved from the NCBI. However, only promoters of three pineapple SERKs could be found which were submitted by our group. Using reciprocal BLAST (BlastP with -evalue $1 \mathrm{e}^{-5}$ ) strategy, we characterized 142 SERK gene family members from 20 species (Figure S3) and obtained their promoter sequences. After a thorough sequence search (BlastN with -evalue 1000 -word_size 7 -gapopen 5 -gapextend 2 -penalty -3 -reward 1), we found that the isolated region is not conserved, but CAAT-box elements are conserved, among the promoters of these genes (Figure S3).

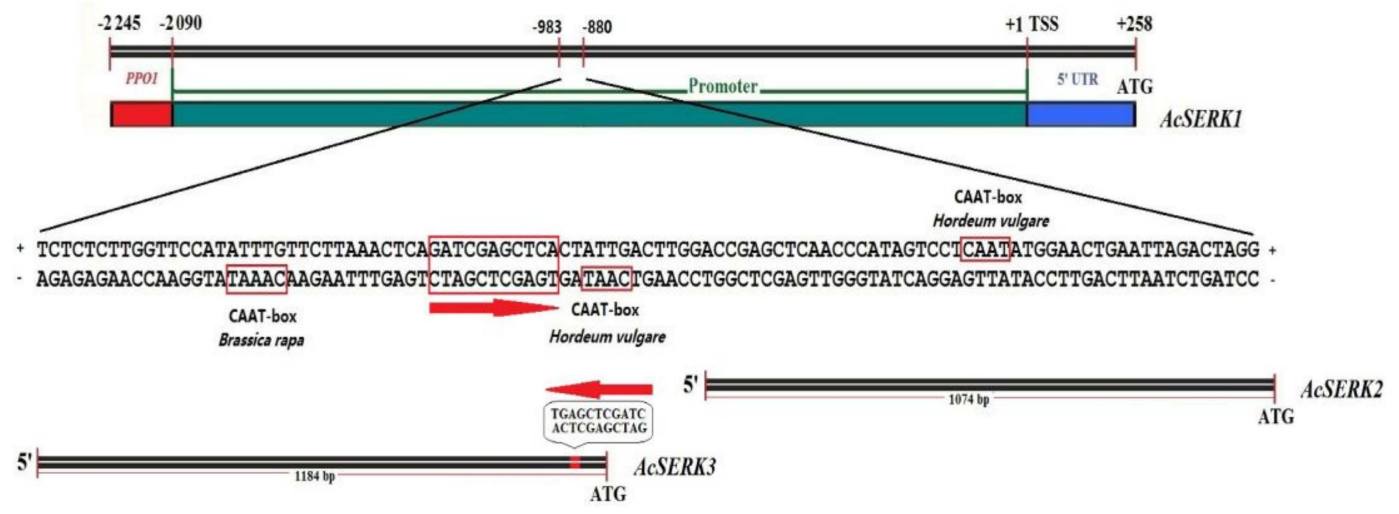

Figure 4. Bioinformatics analysis of embryonic cell-specific sequences.

\section{Discussion}

The expression of genes is regulated by the $5^{\prime}$ upstream regulatory sequence and the transcription factors interacting with it. Therefore, studying the functional regions contained in the $5^{\prime}$ upstream regulatory sequence is of importance for gene regulation study [38,39]. Our findings reveal that the promoter activity of the $5^{\prime}$ regulatory sequence of AcSERK1 gradually increases during the transformation from non-embryonic cells to embryonic cells and then to the formation of globular embryos. In contrast, in the late phase of globular embryo development, promoter activity gradually weakens. This validates the results of our previous study showing the gene expression pattern and promoter activity of AcSERK1 [14,30].

Our previous studies demonstrated that the $5^{\prime}$ regulatory sequence of AcSERK1 has embryonic cell-specific promoter activity under non-stress conditions. However, other researchers have also shown that activity of the $5^{\prime}$ regulatory sequence of AcSERK1 is inducible under dark and low temperature conditions [22]. Thus, identification of the functions of different regions in the $5^{\prime}$ regulatory sequence is essential for studying the corresponding transcriptional regulation and biological function of the protein. Our deletion assay for identifying these cis-acting elements in a regulatory sequence, by monitoring expression of deletion vectors containing GUS or GFP has been widely used [40,41]. The active region required specifically for expression during SE verified here offers an important starting point for the subsequent isolation and identification of embryonic cell-specific elements and the transcription factors interacting with it. Relevant for this, we did identify three predicted CAAT-box elements within the 103 bp sequence of $-983 \mathrm{nt}-880 \mathrm{nt}$. Interestingly, the direction of the embryonic cell-specific region in the AcSERK1 promoter was opposite to the $11 \mathrm{bp}$ homologous sequence in the $5^{\prime}$ upstream regulatory sequence of AcSERK3. Therefore, whether the $11 \mathrm{bp}$ homologous sequence possesses a similar biological function in the $5^{\prime}$ regulatory sequence of AcSERK1 and AcSERK3 also needs further study.

So far, five cases of the $5^{\prime}$ upstream regulatory sequence isolated from the SERK1 gene have been reported. They are the $2000 \mathrm{bp} 5^{\prime}$ upstream regulatory sequence of AtSERK1 isolated from Arabidopsis thaliana [42]; the $2.4 \mathrm{~kb} \mathrm{5'}$ upstream regulatory sequence of OcSERK1 isolated from rice [27]; the $1.5 \mathrm{~kb} 5^{\prime}$ upstream regulatory sequence of MtSERK1 isolated from Medicago truncatula [17]; and the $2348 \mathrm{bp} 5^{\prime}$ upstream regulatory sequence of AcSERK1 from pineapple in our previous study [30]. 
These studies isolated the $5^{\prime}$ upstream regulatory sequences of SERK1 and analyzed its promoter activity in different organs, as well as in somatic embryos, without identification of the different functional regions within them. Compared with the previous studies, our research goes further by validating the cell-specific promoter activity of the AcSERK1 5' upstream regulatory sequence, isolating its functional region by $5^{\prime}$ deletion analysis, and further defining the minimal functional unit of the promoter with a more directed deletion analysis. Although in this study a shorter functional region with embryonic cell-specificity was isolated, the cis-acting element in it was not defined. This would require further deletion analysis and point mutations to identify the conserved sequence of the embryonic cell-specific cis-acting element.

\section{Conclusions}

In summary, SERK1 is a functional gene in the early stage of somatic embryogenesis that likely acts upon the somatic embryogenesis regulatory network. Thus, its upstream transcriptional regulation is of great importance for increasing the rate of somatic embryogenesis. Our isolation and identification of the embryonic cell-specific region within the promoter of AcSERK1 will help to decipher the molecular mechanisms of somatic embryogenesis and thereby improve the efficiency of pineapple SE and in vitro propagation.

Supplementary Materials: The following are available online at http://www.mdpi.com/2073-4425/10/11/883/s1, Figure S1: Structure of AcSERK1 5' upstream regulatory sequence deletion vectors., Figure S2: Structure of AcSERK1 5' upstream regulatory sequence deletion vectors, Figure S3: Distribution of CAAT-box on promoter sequences of 142 SERK family genes from 20 plant species.

Author Contributions: A.L. and Y.H. designed and performed research. A.L. performed most of the experiments and data analysis. A.L., W.L. and C.C. wrote the paper. X.T., X.W., C.L. (Chuhao Li), Q.M., J.X., C.L. (Chaoyang Liu), and Y.D. carried out part of material preparation, RNA extraction. J.H. revised the manuscript. All authors have read and approved the final manuscript.

Funding: The authors gratefully acknowledge editing support from Life Science Editor Helen Pickersgill (Ph.D, Oxford, U.K.). This work was supported by National Natural Science Foundation of China (NO. 31572089), Forming and Regulation of Important Economical Traits in Tropical Crops (NO. 2018YFD1000500) and Central Public-interest Scientific Institution Basal Research Fund for Chinese Academy of Tropical Agricultural Sciences (No. 1630032019016).

Conflicts of Interest: The authors declare no competing financial interests as defined by Nature Research, or other interests that might be perceived to influence the results and/or discussion reported in this paper.

\section{References}

1. Sripaoraya, S.; Marchant, R.; Power, J.B.; Davey, M.R. Plant regeneration by somatic embryogenesis and organogenesis in commercial pineapple (Ananas comosus L.). Vitro. Cell. Dev. Plant 2003, 39, 450-454. [CrossRef]

2. Firoozabady, E.; Gutterson, N. Cost-effective in vitro propagation methods for pineapple. Plant Cell Rep. 2003, 21, 844-850. [PubMed]

3. Gonzalez-Olmedo, J.; Fundora, Z.; Molina, L.J.; Desjardins, Y.; Escalona, M. New contributions to propagation of pineapple (Ananas comosus L. Merr) in temporary immersion bioreactors. Vitro. Cell. Dev. Plant 2005, 41, 87-90. [CrossRef]

4. Mhatre, M. Micropropagation of pineapple, Ananas comosus (L.) Merr. In Protocols for Micropropagation of Woody Trees and Fruits; Springer: Berlin, Germany, 2007; pp. 499-508. [CrossRef]

5. Das, D.; Nirala, N.; Redoy, M.; Sopory, S.; Upadhyaya, K. Encapsulated somatic embryos of grape (Vitis vinifera L.): An efficient way for storage and propagation of pathogen-free plant material. VITIS-J. Grapevine Res. 2015, 45, 179.

6. Gaj, M.D. Factors Influencing Somatic Embryogenesis Induction and Plant Regeneration with Particular Reference to Arabidopsis thaliana (L.) Heynh. Plant Growth Regul. 2004, 43, 27-47. [CrossRef]

7. Teyssier, C.; Maury, S.; Beaufour, M.; Grondin, C.; Delaunay, A.; Metté, C.L.; Ader, K.; Cadene, M.; Label, P. In search of markers for somatic embryo maturation in hybrid larch (Larix $\times$ eurolepis): Global DNA methylation and proteomic analyses. Physiol. Plant. 2013, 150, 271-291. [CrossRef] 
8. Fehér, A. Somatic embryogenesis-Stress-induced remodeling of plant cell fate. BBA Gene Regul. Mech. 2015, 1849, 385-402. [CrossRef]

9. Thompson, D. Challenges for the large-scale propagation of forest trees by somatic embryogenesis-a review. In Proceedings of the Third International Conference of the IUFRO Unit, Vitoria-Gasteiz, Spain, 8-12 September 2014; pp. 81-91.

10. Horstman, A.; Bemer, M.; Boutilier, K. A transcriptional view on somatic embryogenesis. Regeneration 2017, 4, 201-216. [CrossRef]

11. Brandt, B.; Hothorn, M. SERK co-receptor kinases. Curr. Biol. 2016, 26, 225-226. [CrossRef]

12. Fan, M.; Wang, M.; Bai, M.Y. Diverse roles of SERK family genes in plant growth, development and defense response. Sci. China Life Sci. 2016, 59, 889-896. [CrossRef]

13. Hohmann, U.; Lau, K.; Hothorn, M. The Structural Basis of Ligand Perception and Signal Activation by Receptor Kinases. Annu. Rev. Plant Biol. 2017, 68, 109-137. [CrossRef] [PubMed]

14. Ma, J.; He, Y.; Wu, C.; Liu, H.; Hu, Z.; Sun, G. Cloning and Molecular Characterization of a SERK Gene Transcriptionally Induced during Somatic Embryogenesis in Ananas comosus cv. Shenwan. Plant Mol. Biol. Rep. 2012, 30, 195-203. [CrossRef]

15. Rocha, D.I.; Pinto, D.L.; Vieira, L.M.; Tanaka, F.A.; Dornelas, M.C.; Otoni, W.C. Cellular and molecular changes associated with competence acquisition during passion fruit somatic embryogenesis: Ultrastructural characterization and analysis of SERK gene expression. Protoplasma 2016, 253, 595-609. [CrossRef] [PubMed]

16. Hecht, V.; Vielle-Calzada, J.P.; Hartog, M.V.; Boutilier, K.; Grossniklaus, U.; Vries, S.C.D. The Arabidopsis SOMATIC EMBRYOGENESIS RECEPTOR KINASE 1 Gene Is Expressed in Developing Ovules and Embryos and Enhances Embryogenic Competence in Culture. Plant Physiol. 2001, 127, 803-816. [CrossRef] [PubMed]

17. Nolan, K.E.; Irwanto, R.R.; Rose, R.J. Auxin up-regulates MtSERK1 expression in both Medicago truncatula root-forming and embryogenic cultures. Plant Physiol. 2003, 133, 218-230. [CrossRef]

18. Ahmadi, B.; Masoomi-Aladizgeh, F.; Shariatpanahi, M.E.; Azadi, P.; Keshavarz-Alizadeh, M. Molecular characterization and expression analysis of SERK1 and SERK2 in Brassica napus L.: Implication for microspore embryogenesis and plant regeneration. Plant Cell Rep. 2016, 35, 185-193. [CrossRef] [PubMed]

19. Hu, H.; Xiong, L.; Yang, Y. Rice SERK1 gene positively regulates somatic embryogenesis of cultured cell and host defense response against fungal infection. Planta 2005, 222, 107-117. [CrossRef]

20. Chen, H.Y.; Hsieh, E.J.; Cheng, M.C.; Chen, C.Y.; Hwang, S.Y.; Lin, T.P. ORA47 (octadecanoid-responsive AP2/ERF-domain transcription factor 47) regulates jasmonic acid and abscisic acid biosynthesis and signaling through binding to a novel cis-element. New Phytol. 2016, 211, 599-613. [CrossRef]

21. Duan, Y.B.; Li, J.; Qin, R.Y.; Xu, R.F.; Li, H.; Yang, Y.C.; Ma, H.; Li, L.; Wei, P.C.; Yang, J.B. Identification of a regulatory element responsible for salt induction of rice OsRAV2 through ex situ and in situ promoter analysis. Plant Mol. Biol. 2016, 90, 49-62. [CrossRef]

22. Lin, W.Q.; Chen, C.J.; Luan, A.P.; Zhang, J.L.; Feng, J.T.; Zhang, Y.F.; Guo, C.H.; He, Y.H. Transcriptional activity of the $5^{\prime}$ upstream region(-499/+ 258 bp) of AcSERK1. J. Fruit Sci. 2016, 33, 1058-1064.

23. Franssen, H.J.; Kulikova, O.; Willemsen, V.; Heidstra, R. Cis-regulatory PLETHORA promoter elements directing root and nodule expression are conserved between Arabidopsis thaliana and Medicago truncatula. Plant Signal. Behav. 2017, 12, 1278102. [CrossRef] [PubMed]

24. Jiang, L.; Wang, Y.; Zhang, S.; He, R.; Li, W.; Han, J.; Cheng, X.G. Tomato SIDREB1 gene conferred the transcriptional activation of drought-induced gene and an enhanced tolerance of the transgenic Arabidopsis to drought stress. Plant Growth Regul. 2017, 81, 131-145. [CrossRef]

25. Wang, L.W.; He, M.W.; Guo, S.R.; Zhong, M.; Shu, S.; Sun, J. NaCl stress induces CsSAMs gene expression in Cucumis sativus by mediating the binding of CsGT-3b to the GT- 1 element within the CsSAMs promoter. Planta 2017, 245, 889-908. [CrossRef] [PubMed]

26. Baudino, S.; Hansen, S.; Brettschneider, R.; Hecht, V.F.; Dresselhaus, T.; Lörz, H. Molecular characterisation of two novel maize LRR receptor-like kinases, which belong to the SERK gene family. Planta 2001, 213, 1-10. [CrossRef] [PubMed]

27. Ito, Y.; Takaya, K.; Kurata, N. Expression of SERK family receptor-like protein kinase genes in rice. BBA-Gene Struct. Expr. 2005, 1730, 253-258. [CrossRef]

28. Sharma, S.K.; Millam, S.; Hein, I.; Bryan, G.J. Cloning and molecular characterisation of a potato SERK gene transcriptionally induced during initiation of somatic embryogenesis. Planta 2008, 228, 319-330. [CrossRef] 
29. Ma, J.; He, Y.H.; Hu, Z.Y.; Xu, W.T.; Xia, J.X.; Guo, C.H.; Lin, S.Q.; Chen, C.J.; Wu, C.H.; Zhang, J.L. Characterization of the third SERK gene in pineapple (Ananas comosus) and analysis of its expression and autophosphorylation activity in vitro. Genet. Mol. Biol. 2014, 37, 530-539. [CrossRef]

30. Luan, A.P.; He, Y.H.; Lin, W.Q.; Chen, C.J.; Feng, J.T.; Xie, T.; Gong, X.; Xia, J.X. Identification of Transcription Start Site of AcSERK1 and Its Embryospecific Cell Promoter in Pineapple. J. Hortic. 2016, 43, 2251-2256. (In Chinese)

31. Lescot, M.; Déhais, P.; Thijs, G.; Marchal, K.; Moreau, Y.; Van de Peer, Y.; Rouzé, P.; Rombauts, S. PlantCARE, a database of plant cis-acting regulatory elements and a portal to tools for in silico analysis of promoter sequences. Nucleic Acids Res. 2002, 30, 325-327. [CrossRef]

32. He, Y.H.; Luo, J.; Wu, H.T.; Wang, R.X.; Gao, A.P.; Zhao, C.X.; Yu, X.L.; Ye, Z.X.; Wang, Z.H.; Hang, J.Z.; et al. Somatic embryogenesis from leaf base callus of Ananas comosus. J. Fruit Sci. 2007, 24, 59-63. (In Chinese)

33. Gao, Y.; Zan, X.L.; Wu, X.F.; Yao, L.; Chen, Y.L.; Jia, S.W.; Zhao, K.J. Identification of fungus-responsive cis-acting element in the promoter of Brassica juncea chitinase gene, BjCHI1. Plant Sci. 2014, 215, 190-198. [CrossRef] [PubMed]

34. Mariashibu, T.S.; Subramanyam, K.; Arun, M.; Mayavan, S.; Rajesh, M.; Theboral, J.; Manickavasagam, M.; Ganapathi, A. Vacuum infiltration enhances the Agrobacterium-mediated genetic transformation in Indian soybean cultivars. Acta Physiol. Plant. 2013, 35, 41-54. [CrossRef]

35. Jefferson, R.A.; Kavanagh, T.A.; Bevan, M.W. GUS fusions: Beta-glucuronidase as a sensitive and versatile gene fusion marker in higher plants. EMBO J. 1988, 6, 3901-3907. [CrossRef]

36. Wang, H.; Han, J.; Kanagarajan, S.; Lundgren, A.; Brodelius, P.E. Trichome-specific expression of the amorpha-4,11-diene 12-hydroxylase (cyp71av1) gene, encoding a key enzyme of artemisinin biosynthesis in Artemisia annua, as reported by a promoter-gus fusion. Plant Mol. Biol. 2013, 81, 119-138. [CrossRef] [PubMed]

37. Murray, A.; Fu, C.; Habibi, G.; Mcmaster, W.R. Regions in the $3^{\prime}$ untranslated region confer stage-specific expression to the Leishmania mexicana a600-4 gene. Mol. Biol. Parasitol. 2007, 153, 125-132. [CrossRef] [PubMed]

38. Ure, M.E.; Heydari, E.; Pan, W.; Ramesh, A.; Rehman, S.; Morgan, C.; Pinsk, M.; Erickson, R.; Herrmann, J.M.; Dimke, H.; et al. A variant in a Cis-regulatory element enhances claudin-14 expression and is associated with pediatric-onset hypercalciuria and kidney stones. Hum. Mutat. 2017, 38, 649-657. [CrossRef]

39. Villafuerte, B.C.; Barati, M.T.; Rane, M.J.; Isaacs, S.; Li, M.; Wilkey, D.W.; Merchant, M.L. Over-expression of insulin-response element binding protein-1 (IRE-BP1) in mouse pancreatic islets increases expression of RACK1 and TCTPP: Beta cell markers of high glucose sensitivity. BBA Proteins Proteom. 2017, 1865, 186-194. [CrossRef]

40. Nishi-Tatsumi, M.; Yahagi, N.; Takeuchi, Y.; Toya, N.; Takarada, A.; Murayama, Y.; Aita, Y.; Sawada, Y.; Piao, X.; Oya, Y.; et al. A key role of nuclear factor $\mathrm{Y}$ in the refeeding response of fatty acid synthase in adipocytes. FEBS Lett. 2017, 591, 965-978. [CrossRef]

41. Peters, B.; Casey, J.; Aidley, J.; Zohrab, S.; Borg, M.; Twell, D.; Brownfield, L. A Conserved cis-Regulatory Module Determines Germline Fate through Activation of the Transcription Factor DUO1 Promoter. Plant Physiol. 2017, 173, 280-293. [CrossRef]

42. Nolan, K.E. Expression of the Somatic Embryogenesis Receptor-Like Kinase1 (SERK1) gene is associated with developmental change in the life cycle of the model legume Medicago truncatula. J. Exp. Bot. 2009, 60, 1759-1771. [CrossRef]

(C) 2019 by the authors. Licensee MDPI, Basel, Switzerland. This article is an open access article distributed under the terms and conditions of the Creative Commons Attribution (CC BY) license (http://creativecommons.org/licenses/by/4.0/). 